\title{
The Problem of Quasiperiodic Photonic Structures Solved by Considering the Cut of 2D Periodic Structure
}

\author{
Ahmed El Houshy \\ American University in Cairo (AUC), Cairo, Egypt \\ Email: houshy@aucegypt.edu
}

How to cite this paper: El Houshy, A. (2021) The Problem of Quasiperiodic Photonic Structures Solved by Considering the Cut of 2D Periodic Structure. Journal of Applied Mathematics and Physics, 9, 864-888. https://doi.org/10.4236/jamp.2021.95059

Received: March 9, 2021

Accepted: May 14, 2021

Published: May 17, 2021

Copyright $\odot 2021$ by author(s) and Scientific Research Publishing Inc. This work is licensed under the Creative Commons Attribution International License (CC BY 4.0).

http://creativecommons.org/licenses/by/4.0/

\section{(c) (i) Open Access}

\begin{abstract}
The physical objective of solving for eigen-modes of a 1D quasiperiodic structure in photonics has been achieved. This was achieved thru considering this structure as a $1 \mathrm{D}$ projection or cut of a $2 \mathrm{D}$ periodic structure. And the problem is solved in a manner similar to $2 \mathrm{D}$ periodic photonic structures. A mechanical analogy (quasiperiodic orbits) helps to bring conceptual clarity.
\end{abstract}

\section{Keywords}

1D Quasiperiodic Structure, Quasiperiodic Motion, 2D Periodic Structure, Photonics, Mathematical Physics, Eigenvalue Problem, Bloch's Theorem

\section{Introduction}

The idea is to use the construction of a $1 \mathrm{D}$ quasi-periodic structure. The construction uses the diagonal sectioning of a (parent) 2D periodic tiling, the diagonal having an irrational slope (see for example [1]). This construction is used to come up with a (Fourier) series expansion. This series expansion may later be used to solve the EVP (Eigen-Value Problem) Master Equation of Photonics (see [2]). It is found that instead of solving for a single frequency/eigenvalue, we need to solve for two-one for each dimension in the associated 2D periodic tiling problem. It is found that to find the two frequencies, we need to solve the $2 \mathrm{D}$ periodic problem as well as the $1 \mathrm{D}$ quasi-periodic problem. The method of solution and the solution itself closely resembles that of periodic problems to the extent that concepts like reciprocal lattice, periodicity and a Bloch's Theorem still apply (in a modified form for the 1D quasi-periodic problem and exactly for the 2D periodic problem)! A mechanical analogy clarifies matters. 


\subsection{Geometry}

Here is what the 1D quasi-periodic strip looks like (Figure 1(b)):

The Diagonal line or Strip above is a $1 \mathrm{D}$ cut along the $2 \mathrm{D}$ periodic lattice. Regarding the slope of the strip, there are two possibilities:

1) It is rational, and so the resulting $1 \mathrm{D}$ strip is periodic.

2) It is irrational and so the resulting $1 \mathrm{D}$ strip is not periodic. It is quasiperiodic (which in turn is different from a totally random structure.)

The second point basically means that only the bottom left corner vertex of the square is hit by the strip. If the strip hits two vertices, then the unit-cell (marked by the two intersections of the ray with the $2 \mathrm{D}$ periodic structure) is a unit-cell of a 1D structure-as in the rational slope case (Figure 2).

An analogy from mechanics is quite instructive here. A periodic structure is analogous to periodic or cyclic motion, say, closed orbits (librations) or rotations (see [3]). A quasiperiodic structure is analogous to quasiperiodic motion (which in turn is a characteristic of chaotic motion and again different from totally random motion). The following discussion of chaotic motion is based on [3].

The usual orbits in phase space encountered in linear problems are usually deterministic (and linear or periodic) as well as being bounded (confined to a relatively small region of phase space rather than wandering all over the place like space-filling curves or fractals). Examples are the ellipses of the Kepler problem, the circles of the simple harmonic oscillator, and the limit cycle of the van der Pol equation.

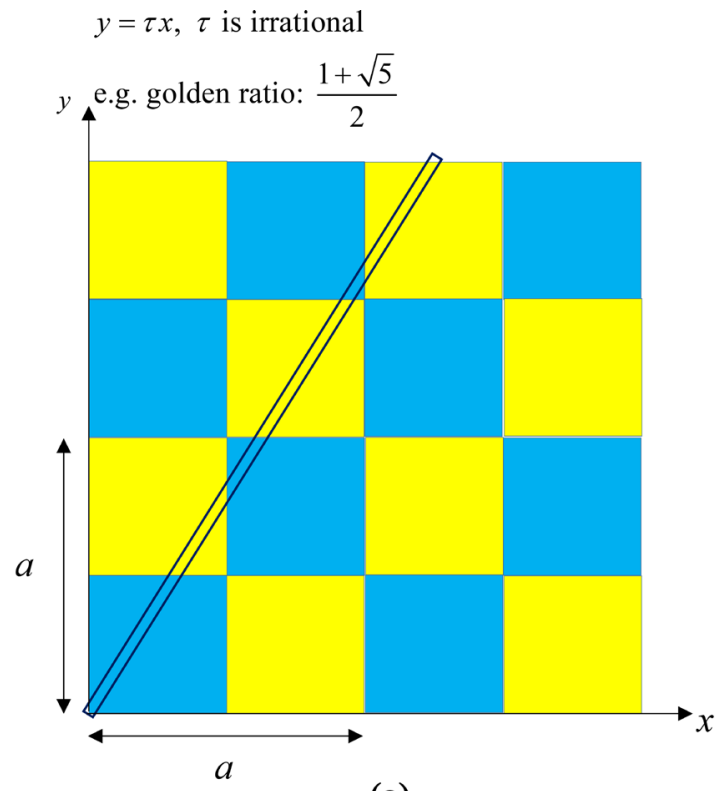

(a)

(b)

Figure 1. (a) It shows how 1D quasiperiodic structure can be constructed out of a 2D periodic structure. This was achieved using a strip with an irrational slope; (b) the strip in Figure 1(a) has been taken out and rotated so that it is horizontal for clarity. 


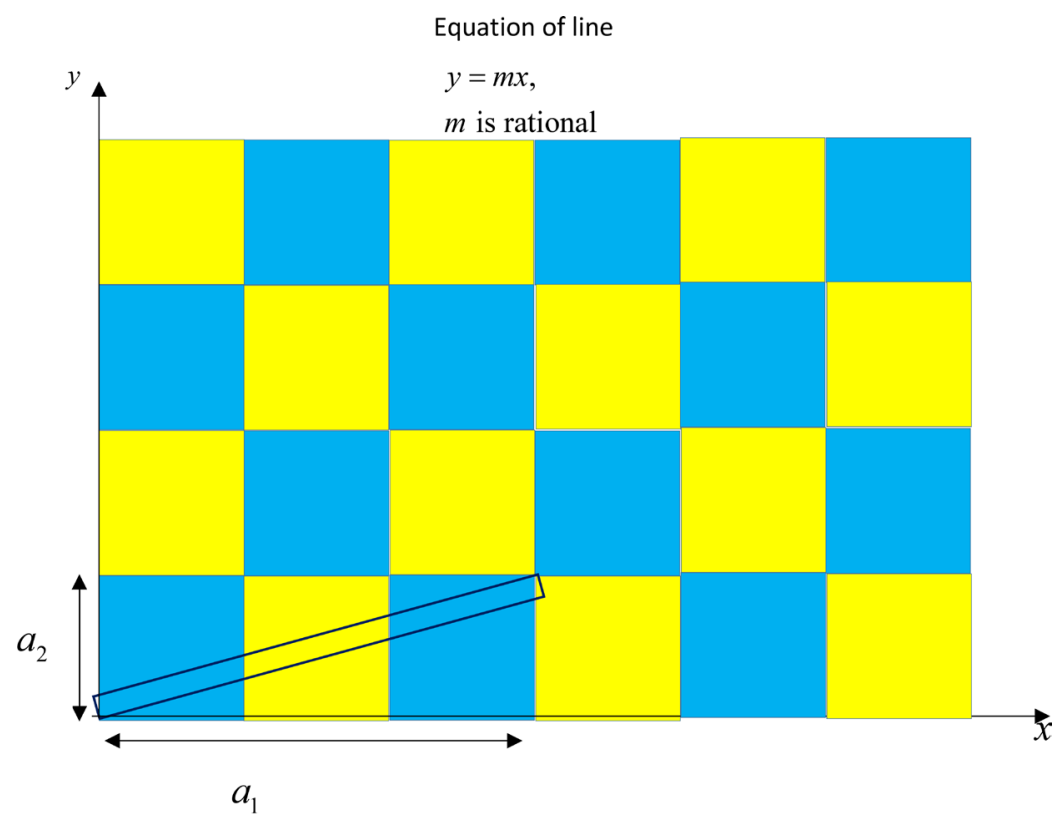

(a)

(b)

Figure 2. (a) this figure shows what happen if the cut has this time a rational slope; (b) Here is what the $1 \mathrm{D}$ unit cell of the periodic strip (as the slope is rational or $1 / 3$ ) looks like.

Under certain conditions, trajectories, called chaotic trajectories, will be encountered in which the motion wanders around an extensive (as opposed to being bounded and confined in a relatively small region)and perhaps irregularly shaped region of phase space in a manner that appears to be random, but that in fact is tempered by constraints.

This path or region where the meandering takes place is an example of a strange attractor. This attractor is different from the usual attractors or repellors encountered for stable and unstable equilibria, respectively. It is called strange because of its (fractal) geometry and chaotic because of its dynamics.

The chaotic trajectory roams here and there, back and forth through this strange attractor region seeming to fill the space (space-filling is a geometric property of fractals and is responsible for fractals having a fractional dimension ... so a trajectory is not your usual $1 \mathrm{D}$ line because it fills a $2 \mathrm{D}$ space and so has a dimension somewhere between 1 and 2), but without ever actually passing through the same point twice (a property reserved for periodic motion). In short, chaotic motion has affinities with ergodic motion-more on this later with characteristics between regular deterministic trajectories and totally random roaming.

\subsection{The Mechanical Analogy}

The motion involved in chaos has the properties of: 
1) Mixing

2) Dense Quasiperiodic orbits

3) Sensitivity to Initial Conditions

Mixing means that if the motion passes through some region in phase space, then it will eventually pass through another region regardless of how far they are apart. A quantification of the idea of the orbit roaming throughout the entire phase space rather than being bounded or confined. A chaotic orbit that visits and revisits that is mixes with all regions of the available phase space is identified with what is called a strange attractor. Its association is not with a localized attractor such as a fixed point or a limit cycle, but rather with a very extended region of phase space, hence the designation strange.

Quasiperiodicorbit means that the orbiting mass repeatedly and irregularly pass through the whole range of the domain without ever closing on themselves, and without any particular time period associated with successive transits. They are dense because they pass through or arbitrarily close to every point of the domain, a property that conforms with the ergodic hypothesis. Like painting or covering a $2 \mathrm{D}$ square piece of canvas with very thin line-like brush strokes.

Sensitivity to Initial conditions: a small change in the initial conditions can result in a large change in position and velocity many transits or iterations later (having discretized the problem and the motion unfolds over discrete time steps). Moreover, this small change (perturbation say) could lead to switching from the linear bounded regime over to the chaotic non-linear one... as two initially close points grow exponentially apart. This exponential growth of the gap is quantified via the Liaponuv exponent, which is directly related to the fractal dimension of the strange attractor. This exponential growth of the gap is just the famous Butterfly Effect-a popular defining property of chaos. An example is a spaceship in an Earth orbit. A small rocket boost will move it to a nearby orbit whereas a strong boost could throw it out of orbit, heading for outer space. Another common example of how linear and chaotic motions differ when periodicity is not present is turbulence in water. While there is streamline flow, two nearby points in the water stay close together as they move along; after the onset of turbulence the same two points, on average, keep moving farther and farther apart. It is worth noting though that, they may now be related by Renormalization owing to fractal nature of turbulent flow. Moreover, renormalization rather than averaging pops up in QFT with its non-linear equations rather than the linear Schrodinger Equation of QM.

\subsubsection{Sensitivity to Initial Conditions, Statistical Mechanics and Quantization}

To drive the point home, observe that this means that Liouville's Theorem which is applied to ensembles in statistical mechanics (the conservation of density of states in phase space) does not hold for chaotic motion. For some initial region $\mathrm{R}, \mathrm{R}$ may deform with time, but number of states within $\mathrm{R}$ is conserved like the constant density of a fluid. That is a system initially inside $\mathrm{R}$ never leaves it and a 
system initially outside $\mathrm{R}$ remains outside it. $\mathrm{R}$ is an exclusive club and its members do not mix with outsiders. This is like continuity equation a flux-density relationship. And it follows from the fact that an element of volume in phase space is an invariant-a Poincare Invariant. However, sensitivity to initial conditions means that if two neigbouring systems/points are initially contained in the same infinitesimal box, later on, they can't. However, their quantized systems would not be sensitive to initial conditions and so Liouville's Theorem and statistical mechanics would still work off equilibrium... and the particles would still be close enough to exchange energies and reach an equilibrium in the first place. This may be thought of as a result of the linearity of Schrodinger's Equation even though the original classical system maybe non-linear (see [4]). (Note that Poisson Bracket Mechanics is a linear PDE in time only while Schrodinger's Equation is linear in both time and space variables)

Liouvilles's Theorem states that the no. of systems within a volume in phase space as well as the volume itself remain constant in time (whence so is the density of systems). The volume may be written as:

$$
V=\oiint \mathrm{d} Q \mathrm{~d} P=\oiint \mathrm{d} q \mathrm{~d} p
$$

Or the volume $V$ is an invariant.

With $P, Q$ being extended or canonical transformations, rather than point transformations, of $p, q, t$ :

$$
\begin{aligned}
& Q=Q(p, q, t) \\
& P=P(p, q, t)
\end{aligned}
$$

However note that in canonical variables:

$$
\oiint \mathrm{d} q \mathrm{~d} p=\Delta p \Delta q
$$

Now, the quantization condition $[p, q]=i \hbar$ leads to (its global version) the uncertainty principle: $\Delta p \Delta q=\frac{\hbar}{2}$; which imposes the invariance of the infinitesimal volume. Ensuring that Liouville's Theorem still applies-even if the quantized system is not linear as in the case of QFT-an improvement on the condition above that required the linearity of the Schrodinger Equation.

That is, quantization condition itself can be thought of as a bounding condition. Which physically makes sense. Quantization of the electron (say in Bohr Model via de Broglie hypothesis: viewing it as a wave rather than a particle) solved the instability problem of the classical atom-seen thru Maxwell's Equations-via removing the secular terms that lead to the instability of the orbit. And quantization of light (viewing it as particles rather than waves) removes the UV divergences-helping the integral have a finite value or making the integral bounded... or a cutoff is introduced. And the whole idea of Renormalizationcentral to all quantum field theories-is again about handling this problem of unboundedness and infinities.

Further discussion and generalizations are available in [4]. 
The property of ergodicity, which involves covering all accessible regions of a domain, is shared by incommensurate non-chaotic orbits with respect to an ordinary attractor (for example, a torus), and by chaotic orbits with respect to a strange attractor. And it is interesting to note that ergodicity is invoked in statistical treatments too.

\subsubsection{Mechanics, Symmetry and Quasiperiodic Functions}

As is well-known, there exists a connection between the existence of additional algebraic constants of the motion, or higher-symmetry groups, and degeneracy in the motions of the system.

In the case of the Kepler and isotropic harmonic oscillator problems, the additional constants of the motion are related to parameters of the orbit. Unless the orbit is closed, that is, the motion is confined to a single curve, we can hardly talk of such orbital parameters. Only when the various components of the motion have commensurate periods will the orbit be closed. The classic example is the two-dimensional anisotropic oscillator. When the frequencies in the $\mathrm{x}$ and $\mathrm{y}$ directions are rational fractions of each other, the particle traverses a closed Lissajous figure. But if the frequencies are incommensurate, the motion of the particle is space-filling or ergodic, eventually coming as close as desired to any specific point in the rectangle defined by the energies of motion in the two directions (ergodic hypothesis). Attempts at finding complicated (and perhaps complex) symmetry groups for incommensurate systems, applicable to all problems of the same number of degrees of freedom, have not yet proved fruitful.

However, if the quasi-periodic trajectory maybe constructed as the projection of a higher-dimensional periodic trajectory ... then the periodicity (of the higher dimensional space) maybe used to solve the problem (so instead of a single $\omega$, breaking it up into two frequencies $\omega_{1}, \omega_{2}$ together with the associated problem maybe the improvement sought). Just as we intend to show for the eigen-value problem of the Master Equation here. The higher dimensional periodicity allows us to reinvoke again Fourier Series and Bloch's Theorem ... and one feels home as he would do with any periodic problem.

Conceptually, the Mechanical Analogue of quasi-periodic motion and quasi-periodic structures helps us understand better what is a quasi-periodic structure and how it differs from a completely random structure. Just as a chaotic system is still deterministic-there is a well defined equation and at times a well defined solution and so is not completely random either.

Moreover, there is a more concrete mathematical advantage to this mechanical analogy. It enables us to see the mathematical form of a quasi-periodic trajectory and hence a quasi-periodic structure. For the position $q$ as a function of time

$$
q(t)=\mathrm{e}^{i\left(\omega_{1}+\omega_{2}\right) t}
$$

With the two frequency incommensurate .... That is,

$$
\frac{\omega_{2}}{\omega_{1}} \notin \mathbb{Q}, \mathbb{Q}=\text { set of rational numbers }
$$


Or the ratio cannot be written as $n / m$ where $n$ and $m$ are integers.

However, note that it took two frequencies to construct one (single-variable) trajectory $q(t)$. Moreover, it is well known that the function $\mathrm{e}^{i \omega t}$ is periodic with the period $T=2 \pi / \omega$. That is, it took two periodic functions (though the periods are incommensurate) to construct one quasi-periodic function. Whence it is natural to expect that a doubly periodic function (periodic in two different dimensions or periodic in two variables e.g. $f(x, y)=\mathrm{e}^{i\left(k_{x} x+k_{y} y\right)}$ encountered when dealing with two dimensional waves) to construct one quasi-periodic function. But a ratio of $y / x=$ irrational needs to be introduced to ensure the incommensurability and this is the role of the cut with the irrational slope.

Again, $y / x=$ irrational (and the slope gets arbitrary close to another vertex of the squares but never actually hits a second vertex) corresponds to an open Lissajou figure. While $y / x=$ rational (leading to two corners being touched by the line giving rise to a periodic system) corresponds to a closed Lissajou figure.

\section{Algebraic Formulation of Cut-Projection Method}

Now, the periodicity of the dielectric 2D square periodic structure $\varepsilon(x, y)$ permits us to expand it in a Fourier Series:

$$
\varepsilon_{\text {periodic }}(x, y)=\sum_{G_{1}, G_{2}} \varepsilon_{G_{1}, G_{2}} \mathrm{e}^{i\left(G_{1} x+G_{2} y\right)}
$$

With $G_{1}, G_{2}$ taking values from a discrete set:

$$
\begin{aligned}
& G_{1}=\frac{2 \pi m}{a}=0, \frac{2 \pi}{a}, \frac{4 \pi}{a}, \cdots \\
& G_{2}=\frac{2 \pi n}{a}=0, \frac{2 \pi}{a}, \frac{4 \pi}{a}, \cdots
\end{aligned}
$$

with $n, m$ being both non-negative integers (but not necessarily equal). While a is the lattice spacing of the unit cell (the tile used to generate the tessellation).

Now how do we algebraically formulate the geometric cut-projection condition? Thru the simple relation, or substitution:

$$
y=\tau x
$$

And so

$$
\varepsilon_{\text {quasiperiodic }}(x, \tau x)=\varepsilon_{\text {quasiperiodic }}(x)=\sum_{G_{1}, G_{2}} \varepsilon_{G_{1}, G_{2}} \mathrm{e}^{i\left(G_{1}+G_{2} \tau\right) x}
$$

Later on, we shall suppress the labels periodic and quasiperiodic when it is clear the function in two variables is $2 \mathrm{D}$ parent periodic and the function is single variable is the $1 \mathrm{D}$ quasi-periodic.

Comparing the harmonics in (2.4) to the one in (1.4), we find that (and making the switch $x \rightarrow t, q(t) \rightarrow \varepsilon(x))$ :

$$
\begin{aligned}
& \omega_{1}=G_{1} \\
& \omega_{2}=G_{2} \tau
\end{aligned}
$$

Moreover observe that: 


$$
\frac{G_{2} \tau}{G_{1}}=\frac{\tau n \cdot 2 \pi / a}{m \cdot 2 \pi / a}=\tau \frac{n}{m}
$$

And as $n, m$ are integers and $\tau$ is irrational, then

$$
\tau \frac{n}{m}=\text { irrational } \times \frac{\text { integer }}{\text { integer }}=\text { irrational } \times \text { rational }=\text { irrational }
$$

Or,

$$
\begin{aligned}
& \frac{G_{2} \tau}{G_{1}}=\text { irrational, just as } \\
& \frac{\omega_{2}}{\omega_{1}}=\text { irrational }
\end{aligned}
$$

And so the identification in (2.5), between a quasi-periodic structure in space and a quasi-periodic trajectory or motion in time, does indeed make sense; and we may express a quasi-periodic structure or function as a Fourier Series as long as the wave-numbers (rather than the frequencies as for quasi-periodic motion) are incommensurate. It might be of interest to compare the harmonics in (2.4) to the form of a quasiperiodic function used to represent the $2 \mathrm{D}$ cut in Crystallography (see [5]):

$$
f(x)=\sin x+\sin \sqrt{2} x
$$

(2.9) Again, $\sin (x)$ alone would be periodic with a period of $2 \pi$. While $\sin (\sqrt{2} x)$ alone would have a period of $2 \pi / \sqrt{2}$. And Cahn says, see [5], that (2.9) is a cut along $f(x)=\sin x+\sin y$ with $y=\sqrt{2} x$.

However the two together could only have a common period if there is a Lowest Common Multiple of the two periods. But this requires that they be commensurate; this is not made possible due to the irrationality of $\tau$. This will be made clearer when considering the example of two different planets orbiting the same star and trying to find when they will be realigned again with the central star.

\section{Similarity of My Equation to Electron Density for Quasi-Crystals}

Also note that if substitute (2.2) in (2.4) we get:

$$
\varepsilon_{\text {quasiperiodic }}(x, \tau x)=\sum_{m, n} \varepsilon_{m, n} \mathrm{e}^{i 2 \pi(m+n \tau) x / a}
$$

Now take the real part:

$$
\operatorname{Re} \varepsilon_{\text {quasiperiodic }}(x, \tau x)=\sum_{m, n} \varepsilon_{m, n} \cos 2 \pi(m+n \tau) x / a
$$

Then projecting the RHS (multiplication of RHS by $\delta_{m n}$ is equivalent to taking the dot product); we get a one-dimensional sum which is equivalent to an expansion of a function in one variable:

$$
\operatorname{Re} \varepsilon_{\text {quasiperiodic }}(x)=\sum_{n} \varepsilon_{n} \cos 2 \pi n(1+\tau) x / a
$$

Which is similar to the form of the electron density for a quasi-crystal in the 
field of Solid State Physics (where quasi-crystals were first experimentally observed; see Chapter 2 in [6]):

$$
\rho(x)=\sum_{n} C_{n} \cos 2 \pi n(1+\tau) x / a
$$

But now $\tau$ is any general irrational number not necessarily the Golden Ratio as in our case. Thus, the expansion we use is in a single variable but in TWO indices stressing the projection of a TWO-dimensional periodic lattice. Unlike the expansion used in Kittle [6], this is just a quasi-periodic expansion in a single index AND a single variable. In essence, the advantage of our expansion is that it allows us to draw analogies with two dimensional periodic problem.

To get a understanding of what the condition (2.3) means, consider again our 2D parent periodic structure. The quasi-periodic structure of direct physical interest lies along the line $y=\tau x$. And so, what we are interested in is the dielectric function $\varepsilon(x, y)$ at the following points (Table 1).

That is, we are sampling the parent $2 \mathrm{D}$ periodic structure along the cut (Figure 3).

Table 1. Some values are shown for the dielectric function to get a better sense of what the condition of irrational slope.

\begin{tabular}{ccc}
\hline$x$ & $y$ & $\varepsilon(x, y)$ \\
\hline 0 & 0 & $\varepsilon(0,0)$ \\
$0.1 a$ & $0.1 a \tau$ & $\varepsilon(0.1 a, 0.1 a \tau)$ \\
1 & $\tau$ & $\varepsilon(1, \tau)$ \\
$a$ & $a \tau$ & $\varepsilon(a, a \tau)$ \\
$2 a$ & $2 a \tau$ & $\varepsilon(2 a, 2 a \tau)$ \\
for a general point, $x_{0}$ & $y_{0}=\tau x_{0}$ & $\varepsilon\left(x_{0}, \tau x_{0}\right)$ \\
\hline
\end{tabular}

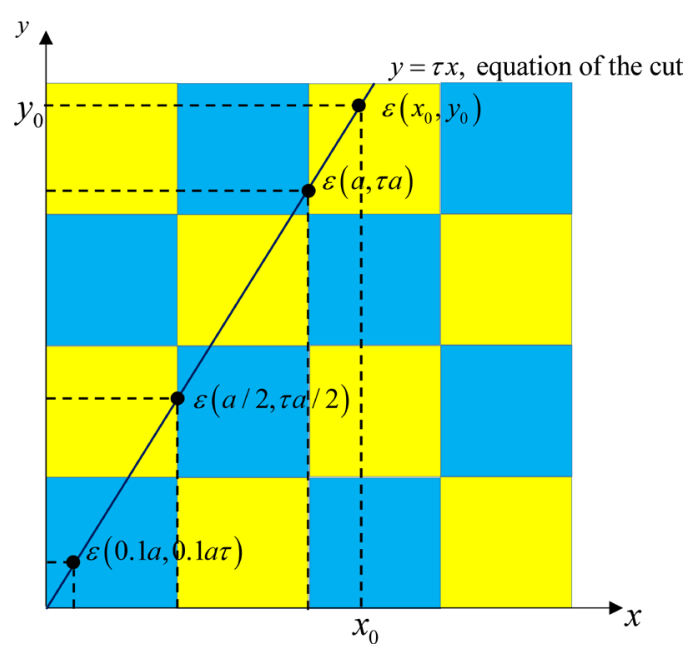

Figure 3. Plotting some of the values in the previous table in order to understand better the meaning of an irrational slope and setting $y=\tau x$. 
And removing the subscript off the general point, shows that indeed the geometric operation of projecting along the cut $y=\tau x$ amounts to an algebraic substitution affecting the transformation:

$$
\varepsilon(x, y) \rightarrow \varepsilon(x, \tau x)=\varepsilon_{\text {quasi }}(x)
$$

If $\varepsilon(x)$ is quasi-periodic so is $F(x)=\frac{1}{\varepsilon(x)}$; since with Dilation Operator $D$ we have:

$$
F(x, y)=\frac{1}{\varepsilon(x, y)}=\frac{1}{\varepsilon(x, \tau x)}=F(x, \tau x)=F_{\text {quasiperiodic }}(x)
$$

Where (2.3) was used to reach the second equality.

\section{Physics}

\subsection{Master Equation in 1D}

$$
\frac{\mathrm{d}}{\mathrm{d} x} \frac{1}{\varepsilon(x)} \frac{\mathrm{d}}{\mathrm{d} x} H(x)=\left(\frac{\omega}{c}\right)^{2} H(x)
$$

NOTE: traditionally in a $1 \mathrm{D}$ periodic multi-layer thin film problem, the propagation direction is taken along the $\mathrm{z}$-axis, which also the direction along which the dielectric varies. That is, we are really solving for $H_{z}=H_{z}(z)$ rather than $H=H(x)$. And the Master Equation should really read:

$$
\frac{\mathrm{d}}{\mathrm{d} z} \frac{1}{\varepsilon(z)} \frac{\mathrm{d}}{\mathrm{d} z} H_{z}(z)=\left(\frac{\omega}{c}\right)^{2} H_{z}(z)
$$

However, we choose to work with the form in (3.1) as it emphasizes the cut-projection correspondence between the $1 \mathrm{D}$ quasi-periodic problem and the $2 \mathrm{D}$ parent periodic problem which is central to this work. Moreover, that neither form affects the final result in reciprocal space, where we have a relation between the wavenumbers, eigenvalues/frequencies and Fourier Coefficients. This is because in Fourier Space, all knowledge of spatial variables of configuration is forgotten thanks to linear independence of the Harmonics.

$$
\text { Now, if } F(x)=\frac{1}{\varepsilon(x)}=\sum_{G_{1}, G_{2}} F_{G} \mathrm{e}^{i\left(G_{1}+G_{2} \tau\right) x}
$$

Where

$$
\begin{aligned}
& \mathrm{e}^{i G_{1} \cdot a}=\mathrm{e}^{i G_{2} \cdot a}=1, \\
& \boldsymbol{G}=\left(G_{1}, G_{2}\right)
\end{aligned}
$$

That is $G_{1}, G_{2}$ are both reciprocal lattices of the square lattice.

And so the 1D quasi-periodic problem is turned into a 2D Periodic Problem.

It is natural to expect a quasi-periodic response:

$$
H(x)=\sum_{k_{1}, k_{2}} C(k) \mathrm{e}^{i\left(k_{1}+k_{2} \tau\right) x}
$$

Now, 


$$
\begin{aligned}
& \frac{\mathrm{d}}{\mathrm{d} x} H(x)=\sum_{k_{1}, k_{2}} i\left(k_{1}+k_{2} \tau\right) C_{k_{1}, k_{2}} \mathrm{e}^{i\left(k_{1}+k_{2} \tau\right) x} \\
& \frac{1}{\varepsilon(x)} \frac{\mathrm{d}}{\mathrm{d} x} H(x)=\sum_{G_{1}, G_{2}} \sum_{k_{1}, k_{2}} i\left(k_{1}+k_{2} \tau\right) F_{G_{1}, G_{2}} C_{k_{1}, k_{2}} \mathrm{e}^{i\left(G_{1}+k_{1}+\left(G_{2}+k_{2}\right) \tau\right) x} \\
& \frac{\mathrm{d}}{\mathrm{d} x} \frac{1}{\varepsilon(x)} \frac{\mathrm{d}}{\mathrm{d} x} H(x) \\
& =-\sum_{G_{1}, G_{2}} \sum_{k_{1}, k_{2}}\left(k_{1}+k_{2} \tau\right)\left(G_{1}+k_{1}+\left(G_{2}+k_{2}\right) \tau\right) F_{G_{1}, G_{2}} C_{k_{1}, k_{2}} \mathrm{e}^{i\left(G_{1}+k_{1}+\left(G_{2}+k_{2}\right) \tau\right) x}
\end{aligned}
$$

And now the Master Equation (3.1) becomes

$$
\begin{aligned}
& -\sum_{G_{1}, G_{2}} \sum_{k_{1}, k_{2}}\left(k_{1}+k_{2} \tau\right)\left(G_{1}+k_{1}+\left(G_{2}+k_{2}\right) \tau\right) F_{G_{1}, G_{2}} C_{k_{1}, k_{2}} \mathrm{e}^{i\left(G_{1}+k_{1}+\left(G_{2}+k_{2}\right) \tau\right) x} \\
& =\left(\frac{\omega}{c}\right)^{2} \sum_{k_{1}, k_{2}} C_{k_{1}, k_{2}} \mathrm{e}^{i\left(k_{1}+k_{2} \tau\right) x}
\end{aligned}
$$

Now the coefficient of $\mathrm{e}^{i\left(k_{1}+k_{2} \tau\right) x}$ on the LHS is (via the transformation $\left.k_{1} \rightarrow k_{1}-G_{1}, k_{2} \rightarrow k_{2}-G_{2}\right)$ :

$$
-\sum_{G_{1}, G_{2}}\left(k_{1}-G_{1}+\left(k_{2}-G_{2}\right) \tau\right)\left(k_{1}+k_{2} \tau\right) F_{G_{1}, G_{2}} C_{k_{1}-G_{1}, k_{2}-G_{2}}
$$

While the coefficient of $\mathrm{e}^{i\left(k_{1}+k_{2} \tau\right) x}$ on the RHS is $\left(\frac{\omega}{c}\right)^{2} C_{k_{1}, k_{2}}$

And now the linear independence of $\mathrm{e}^{i\left(k_{1}+k_{2} \tau\right) x}$ means that its coefficient (due to RHS and LHS) is zero:

$$
\left(\frac{\omega}{c}\right)^{2} C_{k_{1}, k_{2}}+\sum_{G_{1}, G_{2}}\left(k_{1}-G_{1}+\left(k_{2}-G_{2}\right) \tau\right)\left(k_{1}+k_{2} \tau\right) F_{G_{1}, G_{2}} C_{k_{1}-G_{1}, k_{2}-G_{2}}=0
$$

\subsection{Does Bloch Theorem Still Apply?}

The short answer is no. But the periodicity of the parent structure does still have some interesting implications for the quasiperiodic structure (And we still have periodicity in $k$-space).

Following [6], We may first solve (3.9) for the Coefficients of the components of the Field. We may then expand $H_{k}$ in the reciprocal lattice vectors $G_{1}, G_{2}$ of the parent 2D periodic square lattice ( $H_{k}$ the photonic analogue of $\psi_{k}$ in Solid State Physics):

$$
H_{k}(x)=\sum_{G_{1}, G_{2}} C_{k-G} \mathrm{e}^{i\left(k_{1}-G_{1}+\left(k_{2}-G_{2}\right) \tau\right) x}
$$

where $C_{\mathbf{k}-\mathbf{G}}$ is a shorthand for $C_{k_{1}-G_{1}, k_{2}-G_{2}}$ and,

$$
H_{k}(x)=\mathrm{e}^{i\left(k_{1}+k_{2} \tau\right) x} \sum_{G_{1}, G_{2}} C_{k-G} \mathrm{e}^{-i\left(G_{1}+G_{2} \tau\right) x}=\mathrm{e}^{i\left(k_{1}+k_{2} \tau\right) x} u_{k}(x)
$$

where

$$
u_{k}(x)=\sum_{G_{1}, G_{2}} C_{k-G} \mathrm{e}^{-\left(G_{1}+G_{2} \tau\right) x}
$$

Now, translating by the lattice constant of the parent periodic lattice: $x \rightarrow x+a$, 


$$
\begin{aligned}
u_{k}(x+a) & =\sum_{G_{1}, G_{2}} C_{\boldsymbol{k}-\boldsymbol{G}} \mathrm{e}^{-i\left(G_{1}+G_{2} \tau\right)(x+a)} \\
& =\sum_{G_{1}, G_{2}} C_{\boldsymbol{k}-\boldsymbol{G}} \mathrm{e}^{-i\left(G_{1}+G_{2} \tau\right) x} \mathrm{e}^{-i\left(G_{1}+G_{2} \tau\right) a}
\end{aligned}
$$

and,

$$
\mathrm{e}^{-i\left(G_{1}+G_{2} \tau\right) a}=\mathrm{e}^{-i\left(G_{1} a+G_{2} a \tau\right)}=\mathrm{e}^{-i G_{1} a} \mathrm{e}^{-i G_{2} a \tau}=\mathrm{e}^{-i G_{1} a}\left(\mathrm{e}^{-i G_{2} a}\right)^{\tau}
$$

As $G_{1}, G_{2}$ are reciprocal lattice vectors for a periodic square lattice with period a. Thus:

$$
\mathrm{e}^{-i G_{1} a}=1 \text { and } \mathrm{e}^{-i G_{2} a}=1
$$

By definition of a reciprocal lattice vector! That is, had $\tau$ been an integer we would have had $\left(\mathrm{e}^{-i G_{2} a}\right)^{\tau}=1 \quad \ldots$ to handle the problem systematically let's consider the general problem for the slope $p$. The question now is:

$$
\begin{aligned}
& \text { what is the value of }\left(\mathrm{e}^{-i G_{2} a}\right)^{p} \text { ? } \\
& \text { for } \mathrm{e}^{-i G_{2} a}=1
\end{aligned}
$$

And let's consider the following 3 cases:

$$
p=\left\{\begin{array}{c}
\text { integer } \\
\text { rational }=m / n ; m, n \text { integers } \\
\text { irrational }(\text { like } \tau)
\end{array}\right.
$$

For the first case we have:

$$
\left(\mathrm{e}^{-i G_{2} a}\right)^{p}=(1)^{\text {integer }}=1
$$

For the second case

$$
\left(\mathrm{e}^{-i G_{2} a}\right)^{p}=\left(\mathrm{e}^{i 2 \pi r}\right)^{\frac{m}{n}}
$$

where $r$ the set of non-zero integers, by definition of a reciprocal lattice vector. That is $r=\cdots,-2,-1,0,1,2, \cdots$. However $n$ is fixed (as well as $m$ ). Whence, there exists a subset of $r$ which will coincide with the multiples of $n$. Namely, the subset:

$$
R=n r=\cdots,-2 n,-n, 0, n, 2 n, \cdots
$$

And then we end up for this specific subset:

$$
\left(\mathrm{e}^{-i G_{2} a}\right)^{p}=\left(\mathrm{e}^{i 2 \pi R}\right)^{\frac{m}{n}}=\left(\mathrm{e}^{i 2 \pi n r}\right)^{\frac{m}{n}}=\mathrm{e}^{i 2 \pi m r}=1
$$

as now $m, r$ are integers

Meaning we do now have a periodicity na rather than a; as:

$$
\left(\mathrm{e}^{-i G_{2} n a}\right)^{p}=\left(\mathrm{e}^{-i G_{2} n a}\right)^{\frac{m}{n}}=\left(\mathrm{e}^{-i G_{2} a}\right)^{m}=(1)^{m}=1,
$$

as $m$ is an integer

In full we have, for a translation of $u_{k}(x)=\sum_{G_{1}, G_{2}} C_{\boldsymbol{k}-\boldsymbol{G}} \mathrm{e}^{-\left(G_{1}+G_{2} p\right) x}$ by na: 


$$
\begin{aligned}
u_{k}(x+n a) & =\sum_{G_{1}, G_{2}} C_{\boldsymbol{k}-\boldsymbol{G}} \mathrm{e}^{-i\left(G_{1}+G_{2} p\right)(x+n a)} \\
& =\sum_{G_{1}, G_{2}} C_{\boldsymbol{k}-\boldsymbol{G}} \mathrm{e}^{-i\left(G_{1}+G_{2} p\right) x} \mathrm{e}^{-i\left(G_{1}+G_{2} p\right) n a} \\
& =\sum_{G_{1}, G_{2}} C_{\boldsymbol{k}-\boldsymbol{G}} \mathrm{e}^{-i\left(G_{1}+G_{2} p\right) x} \mathrm{e}^{-i G_{1} n a} \mathrm{e}^{-i G_{2} p n a} \\
& =\sum_{G_{1}, G_{2}} C_{\boldsymbol{k}-\boldsymbol{G}} \mathrm{e}^{-i\left(G_{1}+G_{2} p\right) x} \\
& =u_{k}(x)
\end{aligned}
$$

Where in the last step we used the definition of $u_{k}(x)$ for a slope $p$; and in the step before last we used the first of (3.15) and (3.22). Which makes sense, as geometrically: a rational cut with slope $p=m / n$ of a parent periodic structure with period $a$, produces a $1 \mathrm{D}$ structure with period $n a$.

And the physics (eigenfunctions) reflects the geometry.

For the irrational case... well we can't write $p=m / n \quad \ldots$ but we can make successive approximations. For example for the case of $\tau=\frac{1+\sqrt{5}}{2}$, we can make the following decimal approximations with the corresponding fractions (Table 2).

An exact decimal representation would require an infinite no. of decimals places implying an infinite period (or by definition of an irrational number, an exact representation would require the denominator to be an arbitrarily large integer) $\ldots$ or no period as is the case for quasiperiodic systems. And so $u_{k}(x)$ has no period what so ever, and Bloch's Theorem fails.

The lack of a period means that for $p=$ irrational,

$$
\left(\mathrm{e}^{i G_{2} a}\right)^{p} \neq 1
$$

And here the periodicity of the parent lattice kicks in! Explaining why we

Table 2. This table shows the period of the quasi-structure for different approximations of the (irrational) golden-ratio. But since it takes an infinite number of decimal places to accurately describe it; then the resulting period is infinite i.e. there is no periodic representation for the irrational number. And for irrational numbers there is no set of integers that repeat themselves e.g. 1.57575757 . This repetition can be turned into a fractional representation via Summation formula for Geometric Series.

\begin{tabular}{cccc}
\hline No. of decimal places & Decimal approximation & Fractional Approximation m/n & Period na \\
\hline 1 & 1.6 & $16 / 10$ & $10 \mathrm{a}$ \\
2 & 1.62 & $162 / 100$ & $100 \mathrm{a}$ \\
3 & 1.618 & $1618 / 1000$ & $1000 \mathrm{a}$ \\
4 & 1.6180 & $16180 / 10^{4}$ & $10^{4} \mathrm{a}$ \\
5 & 1.61803 & $161803 / 10^{5}$ & $10^{5} \mathrm{a}$ \\
6 & 1.618034 & $1618034 / 10^{6}$ & $10^{6} \mathrm{a}$ \\
7 & 1.6180340 & $16180340 / 10^{7}$ & $10^{7} \mathrm{a}$ \\
8 & 1.61803399 & $161803399 / 10^{8}$ & $10^{8} \mathrm{a}$ \\
\hline
\end{tabular}


might have a Bloch Theorem-usually found for periodic systems for a quasi-periodic system! We have a periodicity with period a. ... An essentially TWO-DIMENSIONAL PERIODICITY that is!

Sub (3.14) in (3.13) to get:

$$
u_{k}(x+a)=\sum_{G_{1}, G_{2}} C_{k-G} \mathrm{e}^{-i\left(G_{1}+G_{2} \tau\right) x}=u(x)
$$

Whence, $u_{k}(x)$ is a periodic function with period a. And combining this result with the form for the field $H_{k}(x)$ (3.11):

$$
H_{k}=u_{k}(x) \mathrm{e}^{i\left(k_{1}+k_{2} \tau\right) x}
$$

That is, the field may be multiplicatively decomposed into a periodic part and a phase factor(responsible for Periodic Boundary Condition ... removed after translating $N$ steps from first unit cell till the last cell... as length of lattice is $L=N a$ ), which is the content of Bloch Theorem for Periodic Systems.

\subsection{Guessing the form of $H(x)$}

To make more sense of the Bloch result above, and understand how 2D periodic systems relate to $1 \mathrm{D}$ quasi-periodic systems... let's take another look at the parent $2 \mathrm{D}$ square lattice:

$$
\varepsilon(x, y)=\varepsilon(x+a, y+a)
$$

And so by Translational Symmetry Considerations (Bloch's Theorem for truly periodic systems), it's natural to expect a system's response after many shifts (for a large integer $N$ ):

$$
H(x, y)=\mathrm{e}^{i\left(k_{1} L+k_{2} L\right)} H(x+N a, y+N a)
$$

That is, every time we translate the field by $L=N a$, it picks up a phase factor $\mathrm{e}^{i\left(k_{1} L+k_{2} L\right)}$

And now the Periodic Boundary Condition gives $H(x, y)=H(x+N a, y+N a)$ :

$$
1=\mathrm{e}^{i\left(k_{1} L+k_{2} L\right)}=\mathrm{e}^{i\left(k_{1} N a+k_{2} N a\right)}=\mathrm{e}^{i k_{1} N a} \mathrm{e}^{i k_{2} N a}
$$

By definition of length $L$ of Periodic Lattice as a large number of unit cells each of length a: $L=N a$

But,

$$
1=\mathrm{e}^{i 2 n_{1} \pi} ; n_{1}=1,2,3, \cdots
$$

Generalizable to:

$$
1=1 \cdot 1=\mathrm{e}^{i 2 n_{1} \pi} \cdot \mathrm{e}^{i 2 n_{2} \pi} ; n_{1}=1,2,3, \cdots ; n_{2}=1,2,3, \cdots
$$

Comparing (3.30) and (3.32) gives:

$$
\begin{aligned}
& \mathrm{e}^{i 2 n_{1} \pi} \cdot \mathrm{e}^{i 2 n_{2} \pi}=\mathrm{e}^{i k_{1} N a} \cdot \mathrm{e}^{i k_{2} N a} \\
& \Rightarrow 2 n_{1} \pi=k_{1} N a \Rightarrow k_{1}=\frac{2 n_{1} \pi}{N a}=\frac{2 \pi}{N a}, \frac{4 \pi}{N a}, \cdots, \frac{2 N \pi}{N a} \\
& \Rightarrow 2 n_{2} \pi=k_{2} N a \Rightarrow k_{2}=\frac{2 n_{2} \pi}{N a}=\frac{2 \pi}{N a}, \frac{4 \pi}{N a}, \cdots, \frac{2 N \pi}{N a}
\end{aligned}
$$


Now the periodicity boundary condition on the field gives:

$$
H(x, y)=H(x+N a, y+N a)
$$

Permitting the Fourier Series Expansion:

$$
\begin{aligned}
& H(x, y)=\sum_{k_{1}, k_{2}} C_{k_{1}, k_{2}} \mathrm{e}^{i\left(k_{1} x+k_{2} y\right)} ; \\
& k_{1}=\frac{2 n_{1} \pi}{N a} ; k_{2}=\frac{2 n_{2} \pi}{N a}
\end{aligned}
$$

Now how do we go from parent periodic lattice $\varepsilon(x, y)$ over to the quasi-periodic lattice $\varepsilon(x)$ ? Thru the following transformation:

$$
y=\tau x
$$

Applying the same transformation to (3.35):

$$
H(x, \tau x)=H(x)=\sum_{k_{1}, k_{2}} C_{k_{1}, k_{2}} \mathrm{e}^{i\left(k_{1}+k_{2} \tau\right) x}
$$

Which the form we used above in (3.5). And now it is clearer why is it that translating the field by a lattice constant $a$ means the field picks up a phase factor without changing form (Bloch's Theorem). This is something inherited from the parent periodic-lattice. Memory of periodicity is retained. That the field and the lattice have the same functional form simplifies the Algebra as it allows "recombination" of the Fourier terms, allowing us to invoke linear dependence to have a system of linear equations in the coefficients, see the steps leading to (3.9).

\subsection{A Clearer Conceptual Explanation of the Failure of Bloch Theorem}

Now we know from the periodic case for the $2 \mathrm{D}$ parent lattice that Bloch's Theorem Applies:

$$
H_{k}(x, y)=u_{k}(x, y) \mathrm{e}^{i\left(k_{1} x+k_{2} y\right)} \text { with } u_{k}(x, y)=u_{k}(x+N a, y+N a)
$$

Applying the same Geometric Transformation $y=\tau x$ (3.36) that took a diagonal section along the $2 \mathrm{D}$ periodic lattice to go over to the $1 \mathrm{D}$ quasi-periodic lattice:

$$
H_{k}(x, y) \rightarrow H_{k}(x, \tau x)=H_{k}(x)=u_{k}(x) \mathrm{e}^{i\left(k_{1}+k_{2} \tau\right) x} \text { with } u_{k}(x, y) \rightarrow u_{k}(x)
$$

And it was verified above that $u_{k}(x)$ is indeed periodic with period $a$.

But now it is much clearer how the Bloch Theorem for the quasi-periodic problem related to the Bloch Theorem of the periodic problem; through the diagonal sectioning transformation (3.36).

And now it is much clearer where the periodicity pops up in a quasi-periodic problem; the physical field $H(x)$ of the quasi-periodic problem inherits the periodicity from the physical field $H(x, y)$ from the parent periodic lattice. Just as the quasi-periodic structure $\varepsilon(x)$ inherits its periodicity from the parent $2 \mathrm{D}$ lattice $\varepsilon(x, y)$.

We may see now how the form of the physical field relates to the geometry of the photonic structure. And how the relation of the parent periodic lattice to the quasi-periodic structure affects the field in the latter. That is, the same relation 
exists between the field $H(x, y)$ in the parent periodic structure and the field $H(x)$ in the quasi-periodic structure: $y=\tau x$.

\subsection{Quasiperiodic and Parent-Periodic Structures - A Comparison}

In $2 \mathrm{D}$ we have a natural decoupling into $\mathrm{TM}\left\{E_{x}, E_{y}, H_{z}\right\}$ and $\mathrm{TE}\left\{H_{x}, H_{y}, E_{z}\right\}$ modes with the governing Equations (based on [7]) (Table 3):

$$
\begin{aligned}
& \Theta H_{z}=\left(\frac{\omega}{c}\right)^{2} H_{z}, \Theta=-\left[\frac{\partial}{\partial x} \frac{1}{\varepsilon(x, y)} \frac{\partial}{\partial x}+\frac{\partial}{\partial y} \frac{1}{\varepsilon(x, y)} \frac{\partial}{\partial y}\right] \\
& \Theta^{\prime} E_{z}=\left(\frac{\omega}{c}\right)^{2} E_{z}, \Theta^{\prime}=-\frac{1}{\varepsilon(x, y)}\left[\frac{\partial^{2}}{\partial x^{2}}+\frac{\partial^{2}}{\partial y^{2}}\right]
\end{aligned}
$$

where,

$$
\begin{aligned}
& H_{z}=H_{z}(x, y), \quad E_{z}=E_{z}(x, y), \\
& i \omega_{0} \varepsilon_{0} \varepsilon(x, y) E_{x}=\frac{\partial H_{z}}{\partial y}, \quad i \omega_{0} \mu_{0} H_{x}=\frac{\partial E_{z}}{\partial y} \\
& -i \omega_{0} \varepsilon_{0} \varepsilon(x, y) E_{y}=\frac{\partial H_{z}}{\partial x}, \quad-i \omega_{0} \mu_{0} H_{y}=\frac{\partial E_{z}}{\partial x}
\end{aligned}
$$

\begin{tabular}{|c|c|c|c|}
\hline & Periodic & & Quasiperiodic \\
\hline Dielectric & $\varepsilon(x, y)=\sum_{G_{1}, G_{2}} \varepsilon_{G_{1}, G_{2}} \mathrm{e}^{i\left(G_{1} x+G_{2} y\right)}$ & $\stackrel{y=\tau x}{\longrightarrow}$ & $\begin{array}{l}\varepsilon(x, \tau x)=\sum_{G_{1}, G_{2}} \varepsilon_{G_{1}, G_{2}} \mathrm{e}^{i\left(G_{1}+G_{2} \tau\right) x} \\
\text { (Projection of Periodic Case) }\end{array}$ \\
\hline Eigenfunction $\mathrm{Hz}$ & $\mathrm{e}^{i\left(k_{1} x+k_{2} y\right)}$ & & $\mathrm{e}^{i\left(k_{1}+k_{2} \tau\right) x} \quad$ (Again, Projection of Periodic Case) \\
\hline $\begin{array}{l}\text { Periodicity in } \\
\text { Configuration/Physical Space }\end{array}$ & Yes & & No \\
\hline Periodicity in Reciprocal Space & Yes & & Yes \\
\hline \multirow[t]{5}{*}{ Bloch Theorem } & Applies & & $\begin{array}{l}\text { Does Not Apply, (due to } 3^{\text {rd }} \text { point above) Chaos and } \\
\text { renormalization on the circle }\end{array}$ \\
\hline & \multirow[t]{4}{*}{$\begin{array}{l}\text { Geometric Periodicity } \\
=>\text { Eigenfunction Periodicity }\end{array}$} & & $\begin{array}{l}\mathrm{e}^{i G_{2}: \text { slope }(x+a)}=\mathrm{e}^{i G_{2}: \text { slope: } x}, \text { Requires: } \\
\left(\mathrm{e}^{i G_{2}: \text { slope } a}\right)=\left(\mathrm{e}^{i G_{2} a}\right)^{\text {slope }}=(1)^{\text {slope }}=1 \\
\text { And the last step requires that } \\
\text { slope }=\text { integer or rational (with adjusted periodicity) } \\
\text { otherwise multivaluedness of roots of unity kicks in } \\
\text { and in our case slope }=\tau=\text { irrational }\end{array}$ \\
\hline & & & $\begin{array}{l}\text { But we still have a relation between Geometry and } \\
\text { Physics(and in fact quasiperiodic periodic problem needs to } \\
\text { be solved in conjunction with periodic problem two } \\
\text { unknown frequencies require two equations see below): }\end{array}$ \\
\hline & & & Projective Periodicity of Geometry \\
\hline & & & $=>$ Projective Periodicity of Eigenfunction \\
\hline Temporal Dependence & $H(x, y ; t)=H(x, y) \mathrm{e}^{-i \omega t}=H(x, y) \mathrm{e}^{-i\left(\omega_{1}+\omega_{2}\right) t}$ & & $H(x ; t)=H(x) \mathrm{e}^{-i\left(\omega_{1}+\omega_{2} \tau\right) t}$ \\
\hline Mechanical Analogy & $\begin{array}{l}\text { Isotropic Oscillator } \\
\text { Or Anisotropic Oscillator (commensurate } \\
\text { case) }\end{array}$ & & Anisotropic Oscillator(incommensurate case) \\
\hline
\end{tabular}

Table 3. This table compares $2 \mathrm{D}$ periodic structure and $1 \mathrm{D}$ quasi periodic structure. 
That is, once we solve for $H_{z}, E_{z}$ we automatically know the remaining components of the TM, TE mode respectively.

We note that thankfully, the two equations in (3.40) are scalar ... and for the case of the TM mode, we have the same scalar unknown as the $1 \mathrm{D}$ case which was also really solving for $H_{z}$, see note at the beginning of the Physics section. And so it is this TM mode that is fit for comparison.

We now use (3.40) to solve the 2D parent periodic problem. We make the following Fourier Expansions:

$$
\begin{aligned}
& F(x, y)=\frac{1}{\varepsilon(x, y)}=\sum_{G_{1}, G_{2}} F_{G} \mathrm{e}^{i\left(G_{1} x+G_{2} y\right)} \\
& H_{z}(x, y)=\sum_{k_{1}, k_{2}} C(k) \mathrm{e}^{i\left(k_{1} x+k_{2} y\right)}
\end{aligned}
$$

Which is similar to the expansions in (3.3) and (3.5) and in fact we are summing over the same wave numbers: $G_{1}, G_{2} ; k_{1}, k_{2}$.

We now substitute (3.42) in the first of (3.40). First note:

$$
\begin{aligned}
& \frac{\partial}{\partial x} \frac{1}{\varepsilon(x, y)} \frac{\partial}{\partial x} H_{z} \\
& =\frac{\partial}{\partial x} \sum_{G_{1}, G_{2}} F_{G} \mathrm{e}^{i\left(G_{1} x+G_{2} y\right)} \frac{\partial}{\partial x} \sum_{k_{1}, k_{2}} C(k) \mathrm{e}^{i\left(k_{1} x+k_{2} y\right)} \\
& =\frac{\partial}{\partial x} \sum_{G_{1}, G_{2}} F_{G} \mathrm{e}^{i\left(G_{1} x+G_{2} y\right)} \sum_{k_{1}, k_{2}} C(k) i k_{1} \mathrm{e}^{i\left(k_{1} x+k_{2} y\right)} \\
& =\frac{\partial}{\partial x} \sum_{G_{1}, G_{2}, k_{1}, k_{2}} F_{\boldsymbol{G}} C(\boldsymbol{k}) i k_{1} \mathrm{e}^{i\left(\left(k_{1}+G_{1}\right) x+\left(k_{2}+G_{2}\right) y\right)} \\
& =\sum_{G_{1}, G_{2}, k_{1}, k_{2}}-k_{1}\left(k_{1}+G_{1}\right) F_{\boldsymbol{G}} C(\boldsymbol{k}) \mathrm{e}^{i\left(\left(k_{1}+G_{1}\right) x+\left(k_{2}+G_{2}\right) y\right)}
\end{aligned}
$$

Similarly,

$$
\frac{\partial}{\partial y} \frac{1}{\varepsilon(x, y)} \frac{\partial}{\partial y} H_{z}=\sum_{G_{1}, G_{2}, k_{1}, k_{2}}-k_{2}\left(k_{2}+G_{2}\right) F_{\boldsymbol{G}} C(\boldsymbol{k}) \mathrm{e}^{i\left(\left(k_{1}+G_{1}\right) x+\left(k_{2}+G_{2}\right) y\right)}
$$

Whence, (3.40) becomes:

$$
\begin{aligned}
& \sum_{G_{1}, G_{2}, k_{1}, k_{2}}\left(k_{1}\left(k_{1}+G_{1}\right)+k_{2}\left(k_{2}+G_{2}\right)\right) F_{\boldsymbol{G}} C(\boldsymbol{k}) \mathrm{e}^{i\left(\left(k_{1}+G_{1}\right) x+\left(k_{2}+G_{2}\right) y\right)} \\
= & \sum_{k_{1}, k_{2}} C(\boldsymbol{k}) \mathrm{e}^{i\left(k_{1} x+k_{2} y\right)}
\end{aligned}
$$

Now the coefficient of $\mathrm{e}^{i\left(k_{1} x+k_{2} y\right)}$ on the LHS is (via the transformation $\left.k_{1} \rightarrow k_{1}-G_{1}, k_{2} \rightarrow k_{2}-G_{2}\right):$

$$
\sum_{G_{1}, G_{2}} k_{1}\left(k_{1}-G_{1}\right)+k_{2}\left(k_{2}-G_{2}\right) F_{G_{1}, G_{2}} C_{k_{1}-G_{1}, k_{2}-G_{2}}
$$

Now the coefficient of $\mathrm{e}^{i\left(k_{1} x+k_{2} y\right)}$ on the RHS is $\left(\frac{\omega}{c}\right)^{2} C_{k_{1}, k_{2}}$

And now the linear independence of $\mathrm{e}^{i\left(k_{1} x+k_{2} y\right)}$ means that its coefficient (due to RHS and LHS) is zero: 


$$
\left(\frac{\omega_{\text {periodic }}}{c}\right)^{2} C_{k_{1}, k_{2}}-\sum_{G_{1}, G_{2}}\left(k_{1}\left(k_{1}-G_{1}\right)+k_{2}\left(k_{2}-G_{2}\right)\right) F_{G_{1}, G_{2}} C_{k_{1}-G_{1}, k_{2}-G_{2}}=0
$$

Comparing this to the analogous result (3.9) of 1D quasiperiodic structure:

$$
\left(\frac{\omega_{\text {quasi }}}{c}\right)^{2} C_{k_{1}, k_{2}}+\sum_{G_{1}, G_{2}}\left(k_{1}-G_{1}+\left(k_{2}-G_{2}\right) \tau\right)\left(k_{1}+k_{2} \tau\right) F_{G_{1}, G_{2}} C_{k_{1}-G_{1}, k_{2}-G_{2}}=0
$$

And so we may use the solution of periodic problem (where we already calculate the Fourier Coefficients $\mathrm{F}$ and solve for the coefficients $\mathrm{C}$ rather than just stop at finding the eigenvalues, that is we solve for the eigenvalues and the eigenvectors) to find the solution of the quasi-periodic problem (same $k_{1}, k_{2}$ and $G_{1}, G_{2}$ and identical F's and C's)

\subsection{Meaning of having a Parent Periodic Structure}

Symmetries (Translational and otherwise) in Reciprocal Space and implied symmetries in the Brillouin Zone (Figure 4).

Condition for Reciprocal lattice vectors:

$$
\boldsymbol{G}_{i} \cdot \boldsymbol{a}_{j}=2 \pi \delta_{i j}
$$

We can't immediately apply the standard equation for 3D lattices as that involves a cross-product that may lead to having a reciprocal lattice vector along $\mathrm{z}$-axis.

Instead we find by inspection that:

$$
\begin{aligned}
& G_{1}=\frac{2 \pi}{\left|a_{1}\right|^{2}} a_{1}=\frac{2 \pi}{a} \hat{\boldsymbol{x}} \\
& \boldsymbol{G}_{2}=\frac{2 \pi}{\left|\boldsymbol{a}_{1}\right|^{2}} \boldsymbol{a}_{2}=\frac{2 \pi}{a} \hat{\boldsymbol{y}}
\end{aligned}
$$

\subsection{Propagation}

First, it is instructive to observe how a wave in $2 \mathrm{D}$ would propagate in Cartesian
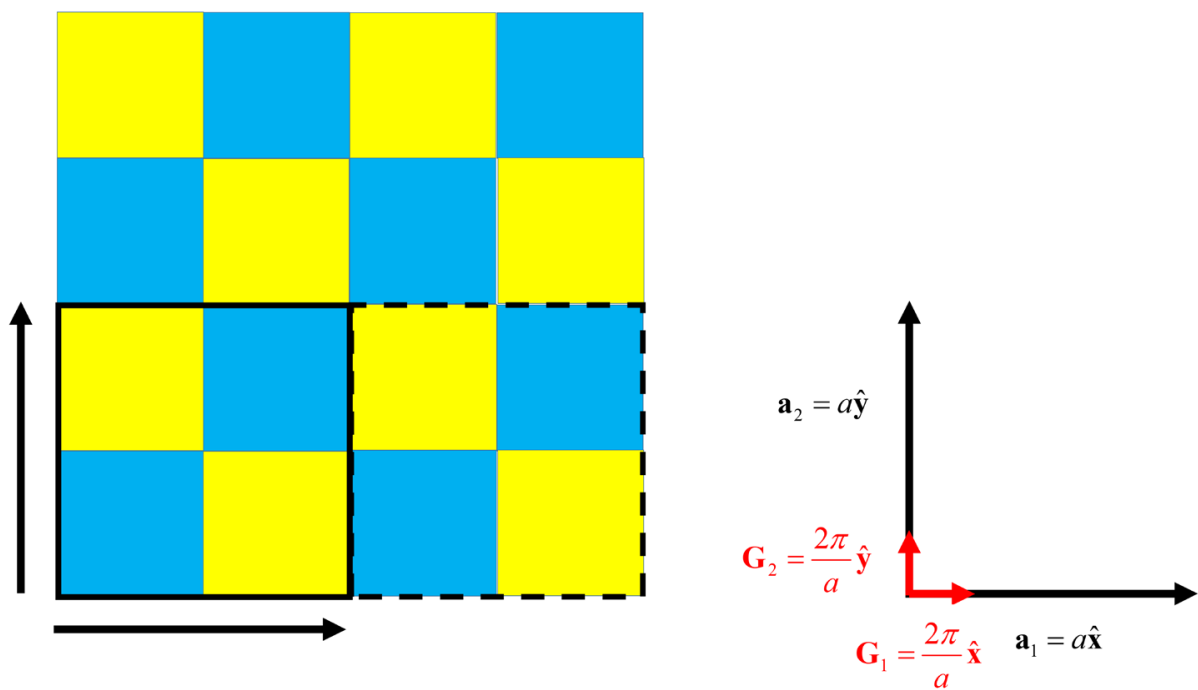

Figure 4. This figure shows the reciprocal lattice vector for a specific $2 \mathrm{D}$ unit cell. 
co-ordinates $\boldsymbol{x}=(x, y)$, without change in form i.e. via a pure translation.

At $t=0$

$$
f(\boldsymbol{x})=\mathrm{e}^{i\left(k_{1} x+k_{2} y\right)}
$$

Changing without form means:

$$
\begin{aligned}
& \boldsymbol{x} \rightarrow \boldsymbol{x}-\boldsymbol{v} t \\
& \boldsymbol{v}=\left(v_{1}, v_{2}\right)
\end{aligned}
$$

And so,

$$
\boldsymbol{x}-\boldsymbol{v} t=(x, y)-\left(v_{1}, v_{2}\right) t=\left(x-v_{1} t, y-v_{2} t\right)
$$

Whence,

$$
\begin{aligned}
f(\boldsymbol{x}-\boldsymbol{v} t) & =\mathrm{e}^{i\left(k_{1}\left(x-v_{1} t\right)+k_{2}\left(y-v_{2} t\right)\right)} \\
& =\mathrm{e}^{i\left(k_{1} x+k_{2} y\right)-i\left(k_{1} v_{1}+k_{2} v_{2}\right) t} \\
& =\mathrm{e}^{i\left(k_{1} x+k_{2} y\right)-i\left(\omega_{1}+\omega_{2}\right) t} \\
& =\mathrm{e}^{i\left(k_{1} x+k_{2} y\right)-i \omega t}
\end{aligned}
$$

Where we have used the relations

$$
\begin{aligned}
& \omega_{i}=k_{i} v_{i} ; \text { for } i=1,2 \\
& \omega=\omega_{1}+\omega_{2}=k_{1} v_{1}+k_{2} v_{2}=\boldsymbol{k} \cdot \boldsymbol{v}, \\
& \text { with the group velocity } \frac{\partial \omega}{\partial \boldsymbol{k}}=\boldsymbol{v}
\end{aligned}
$$

Going thru a similar process for the quasiperiodic case we have:

At $t=0$

$$
f(x)=\mathrm{e}^{i\left(k_{1}+k_{2} \tau\right) x}
$$

moving without change in form means:

$$
x \rightarrow x-c t,
$$

Whence,

$$
\begin{aligned}
f(x-c t) & =\mathrm{e}^{i\left(k_{1}+k_{2} \tau\right)(x-c t)} \\
& =\mathrm{e}^{i\left(k_{1}+k_{2} \tau\right) x-i\left(k_{1} c+k_{2} c \tau\right) t} \\
& =\mathrm{e}^{i\left(k_{1}+k_{2} \tau\right) x-i\left(\omega_{1}+\omega_{2} \tau\right) t}
\end{aligned}
$$

Where we have used the relations

$$
\begin{aligned}
& \omega_{i}=k_{i} c ; \text { for } i=1,2 \\
& \omega=\omega_{1}+\omega_{2} \tau=k_{1} c+k_{2} c \tau
\end{aligned}
$$

This $\omega$ used in separation of time dependence: $H(x, t)=H(x) \mathrm{e}^{-i \omega t}$

(And then we may be having a propagation at a speed faster than $c$...

this is a question to be answered in future research)

It's noteworthy that the temporal part of the last of (3.58) has the same form as quasiperiodic oscillations in mechanics see (1.4) together with the condition (1.5).

However, note that then we run into a contradiction due to the second of 
(3.59). The time dependence for the quasiperiodic case was separated under the assumption:

$$
H(x, t)=H(x) \mathrm{e}^{-i \omega t}
$$

Meaning that the time dependence was seen as Harmonic: periodic as is the case for an SHO (Simple Harmonic Oscillator). However, we found that the spatial dependence of $H(x)=\sum_{k_{1}, k_{2}} C_{k_{1}, k_{2}} \mathrm{e}^{i\left(k_{1}+k_{2} \tau\right) x}$

Implies a time dependence of anisotropic harmonic $\mathrm{e}^{-i\left(\omega_{1}+\omega_{2} \tau\right) t}$ as it has the same temporal dependence as that for a mechanical Anisotropic Harmonic Oscillator (AHO), see (1.4) and (1.5) which cannot be rewritten as a Harmonic temporal dependence as the two periods are incommensurate as previously discussed.

A possible way out is to resolve Maxwell's Equations in the quasiperiodic case using the temporal dependence:

$$
H(x, t)=H(x) \mathrm{e}^{-i\left(\omega_{1}+\omega_{2} \tau\right) t}
$$

\subsection{Physical Interpretation of Two Different Frequencies}

The question now that arises is: What is the physical meaning of the AHO temporal dependence of (3.61)? Here again the 2D periodic parent structure analogy comes to the rescue. Let us first consider propagation in free space

We may first interpret the 1D wave propagation case in 1D free space (invariant under translation in $\mathrm{x}$ direction):

$$
\begin{aligned}
H(x, t)=H_{0}(x) \mathrm{e}^{-i \omega t} \\
\Rightarrow \text { a temporal dependence like a 1D Harmonic Oscillator } \\
\left.\quad \text { (having Amplitude } H_{0}(x)\right) \text { Due to invariance under } \\
\quad \text { translation (with some translation vector) }
\end{aligned}
$$

And now the 2D wave propagation in free space (that is invariant under translation in $\mathrm{x}$ and $\mathrm{y}$ directions):

$$
\begin{aligned}
& H(x, y ; t)=H_{0}(x, y) \mathrm{e}^{-i\left(\omega_{1}+\omega_{2}\right) t}=H_{0}(x, y) \mathrm{e}^{-i \omega t} \\
& \Rightarrow \text { a temporal dependence like a 2D Anisotropic Harmonic Oscillator }
\end{aligned}
$$

(having Amplitude $\left.H_{0}(x)\right)$ Due to invariance under translation

(with 2 different translation vectors - different for different directions) (3.63)

which maybe thought of as a single Harmonic Oscillator with

frequency $\omega=\omega_{1}+\omega_{2}$, as the two frequencies are commensurate

the two translation vectors are commensurate.

Where we have used the $2^{\text {nd }}$ of (3.55). The idea is that, owing to the way $x$ and $t$ are related for a Harmonic wave, we cannot really separate $\mathrm{x}$ from $\mathrm{t}$ (much like SR): moving for a time $t$ at velocity $v$ is the same as translating by $v t$, $\boldsymbol{x} \boldsymbol{\rightarrow} \boldsymbol{x}-\boldsymbol{v} t$, see (3.51) to (3.54). Space $\left(x_{1}, x_{2}\right)$ and time are related through the velocities $\left(v_{1}, v_{2}\right)$ (in Special Relativity there is a single velocity, $c$, that of light). And so two different velocities and two different space co-ordinates together 
with two different wavenumbers naturally implies two different frequencies with: $\omega_{1}=k_{1} v_{1}, \omega_{2}=k_{2} v_{2}$, see (3.59)

That is, combining the two frequencies into one

$$
\text { commensurate case : } \omega_{1}+\omega_{2}=\omega
$$

Is natural if the translation in both directions is commensurate, but if they are not? Then combining them does not make sense and it is best to revert to the original natural decomposition.

$$
\text { incommensurate case : } \omega_{1}+\omega_{2} \neq \omega
$$

That is the physical property of incommensurability in frequencies is due to incommensurability in wavenumbers (which in turn is due to the geometric property of quasiperiodicity)... with an effect much like that of having anisotropic harmonic oscillators. With the help of quantum mechanics, we may further elaborate on the harmonic oscillators:

$$
\begin{aligned}
& H(x, t)=H_{0} \mathrm{e}^{-i \omega t} \\
\Rightarrow & \text { a stream of photons with Amplitude } H(x), \\
& \text { with energy like a (quantized) Harmonic Oscillator, } E=\hbar \omega \\
H(x, y ; t)=H_{0} \mathrm{e}^{-i\left(\omega_{1}+\omega_{2}\right) t}=H_{0} \mathrm{e}^{-i \omega t} & \text { a stream of photons with Amplitude } H_{0} \text {, with energy like a 2D } \\
& \text { Anisotropic Harmonic Oscillator with frequencies } \omega_{1}, \omega_{2} \\
& \text { which maybe thought of as a single Simple Harmonic Oscillator } \\
& \text { with frequency } \omega=\omega_{1}+\omega_{2} \text { and energy } E=\hbar \omega
\end{aligned}
$$

While for incommensurable frequencies we have:

$$
\begin{aligned}
& H(x, y ; t)=H_{0}(x, y) \mathrm{e}^{-i\left(\omega_{1}+\omega_{2}\right) t}, \omega_{2} / \omega_{1} \notin \mathbb{Q} \\
& \Rightarrow \text { a stream of photons with Amplitude } H_{0}(x, y) \text {, with energy like } \\
& \quad \text { a 2D Anisotropic Harmonic Oscillator with incommensurable } \\
& \quad \text { frequencies } \omega_{1}, \omega_{2} \text { and energy } E=\hbar\left(\omega_{1}+\omega_{2}\right)
\end{aligned}
$$

With the help of quantum field theory (more precisely QED); the Harmonic Oscillators Analogy is more than a guiding analogy or a pedagogical device ... and instead becomes a physical explanation. And now the incommensurable frequencies are due to a $2 \mathrm{D}$ lattice of harmonic oscillators with a spring constant in the $\mathrm{x}$-direction different from the $\mathrm{y}$-direction. Whence the incommensurability of frequencies is due to Anisotropic Oscillators inseparable from anisotropy of physical properties (spring constants) in space. And it would be interesting to carry out the solution of the quasiperiodic photonics problem in the framework of QED. For now, we will work out the solution in the classical realm of Maxwell's Equations.

\subsection{Solution of Maxwell's Equations with Temporal Dependence $\mathrm{e}^{-i\left(\omega_{1}+\omega_{2} \tau\right) t}$}

The basic idea is that we shall derive the governing Master Equation for incom- 
mensurate frequencies via the assumption

$$
\begin{aligned}
& \boldsymbol{H}(\boldsymbol{x}, t)=\boldsymbol{H}(\boldsymbol{x}) \mathrm{e}^{-i\left(\omega_{1}+\omega_{2} \tau\right) t} \\
& \boldsymbol{E}(\boldsymbol{x}, t)=\boldsymbol{E}(\boldsymbol{x}) \mathrm{e}^{-i\left(\omega_{1}+\omega_{2} \tau\right) t}
\end{aligned}
$$

Rather than the usual assumption (as say is found in [2])

$$
\begin{aligned}
& \boldsymbol{H}(\boldsymbol{x}, t)=\boldsymbol{H}(\boldsymbol{x}) \mathrm{e}^{-i \omega t} \\
& \boldsymbol{E}(\boldsymbol{x}, t)=\boldsymbol{E}(\boldsymbol{x}) \mathrm{e}^{-i \omega t}
\end{aligned}
$$

Starting from Maxwell's Equations:

$$
\begin{aligned}
& \nabla \cdot \boldsymbol{B}=0, \\
& \nabla \cdot \boldsymbol{D}=\rho, \\
& \nabla \times \boldsymbol{E}+\frac{\partial \boldsymbol{B}}{\partial t}=0, \\
& \nabla \times \boldsymbol{H}-\frac{\partial \boldsymbol{D}}{\partial t}=\boldsymbol{J},
\end{aligned}
$$

Now if we restrict ourselves to a medium with the following properties:

- sourceless medium(no charges and no currents: $\rho=0, \boldsymbol{J}=0$ )

- Nonmagnetic $\mu=\mu_{0} \Rightarrow \boldsymbol{B}(\boldsymbol{r})=\mu_{0} \boldsymbol{H}(\boldsymbol{r})$

- Field Strengths are small so that we are in the linear Regime (material is isotropic so dielectric is a vector rather than a tensor) i.e.

$\boldsymbol{D}(\boldsymbol{r}, \omega)=\varepsilon_{0} \varepsilon(\boldsymbol{r}, \omega) \boldsymbol{E}(\boldsymbol{r}, \omega)$

- Furthermore, we ignore any frequency dependence and simply choose appropriate dielectric value for the frequency range of interest.

- And we restrict attention to transparent materials: the dielectric is Real and Positive.

These assumptions reduce Maxwell's Equations to the following:

$$
\begin{aligned}
& \nabla \cdot \boldsymbol{H}(\boldsymbol{r}, t)=0, \\
& \nabla \cdot(\varepsilon(\boldsymbol{r}) \boldsymbol{E}(\boldsymbol{r}, t))=0, \\
& \nabla \times \boldsymbol{E}(\boldsymbol{r}, t)+\mu_{0} \frac{\partial \boldsymbol{H}(\boldsymbol{r}, t)}{\partial t}=0, \\
& \nabla \times \boldsymbol{H}(\boldsymbol{r}, t)-\varepsilon_{0} \varepsilon(\boldsymbol{r}) \frac{\partial \boldsymbol{E}(\boldsymbol{r}, t)}{\partial t}=0,
\end{aligned}
$$

Now here comes the crucial step, regarding the temporal dependence, we will assume the form (3.69) in rather than the usual form (3.70):

$$
\begin{aligned}
& \boldsymbol{H}(\boldsymbol{r}, t)=\boldsymbol{H}(\boldsymbol{r}) \mathrm{e}^{-i\left(\omega_{1}+\omega_{2} \tau\right) t} \Rightarrow \frac{\partial}{\partial t} \boldsymbol{H}(\boldsymbol{r}, t)=-\boldsymbol{H}(\boldsymbol{r}) i\left(\omega_{1}+\omega_{2} \tau\right) \mathrm{e}^{-i\left(\omega_{1}+\omega_{2} \tau\right) t} \\
& \boldsymbol{E}(\boldsymbol{r}, t)=\boldsymbol{E}(\boldsymbol{r}) \mathrm{e}^{-i\left(\omega_{1}+\omega_{2} \tau\right) t} \Rightarrow \frac{\partial}{\partial t} \boldsymbol{E}(\boldsymbol{r}, t)=-\boldsymbol{E}(\boldsymbol{r}) i\left(\omega_{1}+\omega_{2} \tau\right) \mathrm{e}^{-i\left(\omega_{1}+\omega_{2} \tau\right) t}
\end{aligned}
$$

And so the two divergence equations again give:

$$
\begin{aligned}
& \nabla \cdot \boldsymbol{H}(\boldsymbol{r})=0, \\
& \nabla \cdot(\varepsilon(\boldsymbol{r}) \boldsymbol{E}(\boldsymbol{r}))=0,
\end{aligned}
$$

And now the two curl equations become: 


$$
\begin{aligned}
& \nabla \times \boldsymbol{E}(\boldsymbol{r}, t)-i \mu_{0}\left(\omega_{1}+\omega_{2} \tau\right) \boldsymbol{H}(\boldsymbol{r}, t)=0, \\
& \nabla \times \boldsymbol{H}(\boldsymbol{r}, t)+i \varepsilon_{0} \varepsilon(\boldsymbol{r})\left(\omega_{1}+\omega_{2} \tau\right) \boldsymbol{E}(\boldsymbol{r}, t)=0,
\end{aligned}
$$

The $2^{\text {nd }}$ of (3.75) gives:

$$
\frac{1}{\varepsilon(\boldsymbol{r})} \nabla \times \boldsymbol{H}(\boldsymbol{r}, t)=-i \varepsilon_{0}\left(\omega_{1}+\omega_{2} \tau\right) \boldsymbol{E}(\boldsymbol{r}, t),
$$

Taking the curl of (3.76) and using the $1^{\text {st }}$ of (3.75) gives:

$$
\nabla \times \frac{1}{\varepsilon(\boldsymbol{r})} \nabla \times \boldsymbol{H}(\boldsymbol{r}, t)=\varepsilon_{0} \mu_{0}\left(\omega_{1}+\omega_{2} \tau\right)^{2} \boldsymbol{H}(\boldsymbol{r}, t)
$$

And using $1 / c^{2}=\varepsilon_{0} \mu_{0}$

$$
\nabla \times \frac{1}{\varepsilon(\boldsymbol{r})} \nabla \times \boldsymbol{H}(\boldsymbol{r}, t)=\frac{1}{c^{2}}\left(\omega_{1}+\omega_{2} \tau\right)^{2} \boldsymbol{H}(\boldsymbol{r}, t)
$$

The same as the original problem with

$$
\omega \rightarrow \omega_{1}+\omega_{2} \tau
$$

Periodic problem will give eigenvalues:

$$
\frac{1}{c^{2}}\left(\omega_{1}+\omega_{2}\right)^{2}
$$

Two equations with two variables and we may solve for $\omega_{1}, \omega_{2}$

And the $2 \mathrm{D}$ parent problem and the $1 \mathrm{D}$ quasi-periodic problem are inseparable!

Solving the $2 \mathrm{D}$ parent periodic problem, we replace the time dependence in (3.73) by the equation before last in (3.54):

$$
\begin{aligned}
& \boldsymbol{H}(\boldsymbol{r}, t)=\boldsymbol{H}(\boldsymbol{r}) \mathrm{e}^{-i\left(\omega_{1}+\omega_{2}\right) t} \Rightarrow \frac{\partial}{\partial t} \boldsymbol{H}(\boldsymbol{r}, t)=-\boldsymbol{H}(\boldsymbol{r}) i\left(\omega_{1}+\omega_{2} \tau\right) \mathrm{e}^{-i\left(\omega_{1}+\omega_{2}\right) t} \\
& \boldsymbol{E}(\boldsymbol{r}, t)=\boldsymbol{E}(\boldsymbol{r}) \mathrm{e}^{-i\left(\omega_{1}+\omega_{2}\right) t} \Rightarrow \frac{\partial}{\partial t} \boldsymbol{E}(\boldsymbol{r}, t)=-\boldsymbol{E}(\boldsymbol{r}) i\left(\omega_{1}+\omega_{2} \tau\right) \mathrm{e}^{-i\left(\omega_{1}+\omega_{2}\right) t}
\end{aligned}
$$

And so the two divergence equations again give:

$$
\begin{aligned}
& \nabla \cdot \boldsymbol{H}(\boldsymbol{r})=0, \\
& \nabla \cdot(\varepsilon(\boldsymbol{r}) \boldsymbol{E}(\boldsymbol{r}))=0,
\end{aligned}
$$

\subsection{Does Transversality Survive for $E(r)=A \mathrm{e}^{i\left(k_{1}+k_{2}\right) r}$ ?}

And now the two curl equations become:

$$
\begin{aligned}
& \nabla \times \boldsymbol{E}(\boldsymbol{r}, t)-i \mu_{0}\left(\omega_{1}+\omega_{2}\right) \boldsymbol{H}(\boldsymbol{r}, t)=0, \\
& \nabla \times \boldsymbol{H}(\boldsymbol{r}, t)+i \varepsilon_{0} \varepsilon(\boldsymbol{r})\left(\omega_{1}+\omega_{2}\right) \boldsymbol{E}(\boldsymbol{r}, t)=0,
\end{aligned}
$$

The $2^{\text {nd }}$ of (3.75) gives:

$$
\frac{1}{\varepsilon(\boldsymbol{r})} \nabla \times \boldsymbol{H}(\boldsymbol{r}, t)=-i \varepsilon_{0}\left(\omega_{1}+\omega_{2}\right) \boldsymbol{E}(\boldsymbol{r}, t),
$$

Taking the curl of (3.76) and using the $1^{\text {st }}$ of (3.75) gives: 


$$
\nabla \times \frac{1}{\varepsilon(\boldsymbol{r})} \nabla \times \boldsymbol{H}(\boldsymbol{r}, t)=\frac{1}{c^{2}}\left(\omega_{1}+\omega_{2}\right)^{2} \boldsymbol{H}(\boldsymbol{r}, t)
$$

The same as the original problem with

$$
\omega \rightarrow \omega_{1}+\omega_{2}
$$

\section{Conclusions}

The cut procedure of 2D structure has allowed us to solve for the Eigen-Value Problem of Photonics. Moreover, the mechanical analogy (of commensurate and incommensurate orbits) served as a guide. But now it is much clearer how the Bloch Theorem for the quasi-periodic problem related to the Bloch Theorem of the periodic problem, through the diagonal sectioning transformation (3.36).

The periodicity pops up in a quasi-periodic problem thru inheritance: the physical field $H(x)$ of the quasi-periodic problem inherits the periodicity from the physical field $H(x, y)$ from the parent periodic lattice. Just as the quasi-periodic structure $\varepsilon(x)$ inherits its periodicity from the parent 2D lattice $\varepsilon(x, y)$. Similarly, the same relation exists between the field $H(x, y)$ in the parent periodic structure and the field $H(x)$ in the quasi-periodic structure: $y=\tau x$.

The question of physical propagation is trickier and deserves further research.

\section{Acknowledgements}

I would like to thank Dr. Salah El Nahawy, the professor who taught me Solid State Physics (Similar to photonics in many ways e.g. Bloch's Theorem). He also showed us how to solve PDEs using eigen-expansion in Mathematical Physics Course. Moreover, he also taught the author Classical Mechanics making the book by Goldstein accessible and allowing the author to spot the mechanical analogy.

\section{Conflicts of Interest}

The author declares no conflicts of interest regarding the publication of this paper.

\section{References}

[1] Janot, C. (1994) Quasicrystals A Primer. 2nd Ed., Clarendon Express, Oxford. https://doi.org/10.1007/978-3-662-22223-2_9

[2] Joannopoulos, J., et al. (1995) Photonic Crystals Molding Flow of Light. Princeton University Press, Princeton and Oxford.

[3] GoldStein, H., et al. (2000) Classical Mechanics. 3rd Ed., Addison Wesely, San Francisco.

[4] Poulin, D. (2002) A Rough Guide to Quantum Chaos. University of Waterloo, Waterloo.

[5] Cahn, J.W., et al. (1986) Quasi-Periodic Crystals: A Revolution in Crystallography. MRS Bulletin, 11, 9-14. https://doi.org/10.1557/S0883769400069438 
[6] Kittel, C. (1954) Introduction to Solid State Physics. $7^{\text {th }}$ Ed., John Wiley and Sons, Berkeley. https://doi.org/10.1063/1.3061720

[7] Nielsen (2003) Triangular Photonic Band Gap Crystals-Implementation in Air-Guiding Optical Fibers. University of Aarhus, Aarhus. 